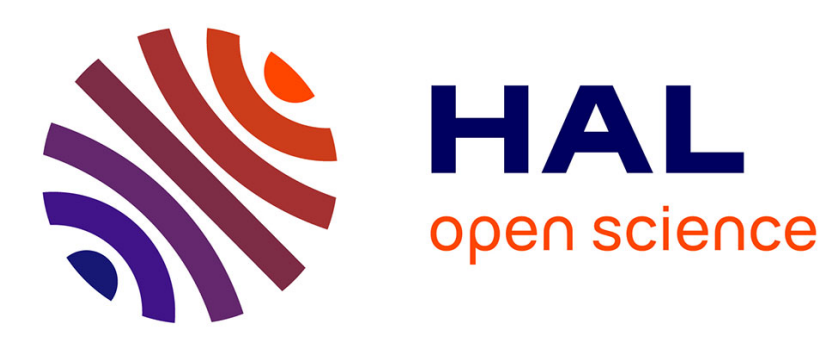

\title{
Exploration of the NH3-H2 van der Waals interaction by high level ab initio calculations
}

M. Mladenovic, Marius Lewerenz, G. Cilpa, P. Rosmus, Gilberte Chambaud

\section{To cite this version:}

M. Mladenovic, Marius Lewerenz, G. Cilpa, P. Rosmus, Gilberte Chambaud. Exploration of the NH3H2 van der Waals interaction by high level ab initio calculations. Chemical Physics, 2008, 346 (1-3), pp.237. 10.1016/j.chemphys.2008.03.004 . hal-00750850

\section{HAL Id: hal-00750850 https://hal.science/hal-00750850}

Submitted on 12 Nov 2012

HAL is a multi-disciplinary open access archive for the deposit and dissemination of scientific research documents, whether they are published or not. The documents may come from teaching and research institutions in France or abroad, or from public or private research centers.
L'archive ouverte pluridisciplinaire HAL, est destinée au dépôt et à la diffusion de documents scientifiques de niveau recherche, publiés ou non, émanant des établissements d'enseignement et de recherche français ou étrangers, des laboratoires publics ou privés. 


\title{
Exploration of the $\mathrm{NH}_{3}-\mathrm{H}_{2}$ van der Waals Interaction by High Level ab initio Calculations
}

\author{
Mirjana Mladenović, ${ }^{*}$ Marius Lewerenz, ${ }^{\dagger}$ Geraldine Cilpa,${ }^{\ddagger}$ Pavel Rosmus,${ }^{\S}$ and Gilberte Chambaud ${ }^{\uparrow}$ \\ Université Paris Est, Marne la Vallée, Laboratoire de Chimie Théorique (LCT), \\ EA 2180, 5 bd Descartes, 77454 Marne la Vallée Cedex 2, France
}

(Dated: September 1, 2008)

\begin{abstract}
The intermolecular potential energy for the van der Waals complex between ammonia and the hydrogen molecule has been studied by means of the coupled cluster $\operatorname{CCSD}(\mathrm{T})$ method and aug-cc-pVXZ $(\mathrm{X}=\mathrm{D}, \mathrm{T}, \mathrm{Q}, 5)$ basis sets and with inclusion of the Boys and Bernardi counterpoise correction. For sufficiently large basis sets the only true electronic minimum energy structure of $\mathrm{NH}_{3}-\mathrm{H}_{2}$ is found to possess $C_{3 v}$ point group symmetry. Various minimum energy paths for the relative motion of $\mathrm{NH}_{3}$ and $\mathrm{H}_{2}$ are analysed in order to understand the topography of the intermolecular potential. The complete basis set limit for the electronic dissociation energy is estimated to be about $253 \mathrm{~cm}^{-1}$ at the $\operatorname{CCSD}(\mathrm{T})$ level.
\end{abstract}

Keywords: Ab initio calculations; $\mathrm{NH}_{3}-\mathrm{H}_{2}$; Potential energy surface; van der Waals complexes; BSSE; Electric properties

\section{INTRODUCTION}

The weak van der Waals interaction of ammonia with $\mathrm{H}_{2}$ has mainly attracted attention due to its implication in inelastic collisions between hydrogen and ammonia molecules leading to rotational excitation of ammonia and the possibility of population inversion. ${ }^{1-3}$ The dynamics of $\mathrm{NH}_{3}-\mathrm{H}_{2}$ is of astrophysical interest in interstellar clouds and the hydrogen rich atmospheres of heavy planets. The existence of nuclear spin isomers in both partners in the complex leads to highly specific symmetry constraints. Matrix isolation studies ${ }^{4,5}$ and gas phase studies ${ }^{6-8}$ of $\mathrm{H}_{2}$ containing complexes indicate a significant influence of the rotational state of $\mathrm{H}_{2}$ for complex formation which leads to marked differences between $o-\mathrm{H}_{2}$ and $p-\mathrm{H}_{2}$.

The intermolecular complex $\mathrm{NH}_{3}-\mathrm{H}_{2}$ should exhibit interesting multidimensional tunnelling dynamics $\left(\mathrm{NH}_{3}\right.$ inversion and internal rotation of $\mathrm{H}_{2}$ ) due to multiple symmetrically equivalent potential energy minima. The bound states of $\mathrm{NH}_{3}-\mathrm{H}_{2}$ are, however, very little known. The high frequency predissociating modes of the related and isoelectronic $\mathrm{HF}-\mathrm{H}_{2}$ and $\mathrm{H}_{2} \mathrm{O}-\mathrm{H}_{2}$ have been observed by Nesbitt and coworkers, ${ }^{6-8}$ but no comparable data exist for ammonia. Theoretical studies of the latter two complexes indicate the possibility of more than one minimum energy structure, $\mathrm{H}_{2}$ acting either as proton acceptor or as proton donor. ${ }^{9}$ These 'isomers' would probably not represent distinct structures due to the large zero point energy but might leave their signature in the internal rotation-tunnelling dynamics. An accurate binary $\mathrm{NH}_{3}-\mathrm{H}_{2}$ potential would be a key element in the analysis of the role of resonances in low energy collisions ${ }^{10}$ and for the theoretical study of the effect of quantum matrices like helium ${ }^{11}$ or $p$-hydrogen ${ }^{12}$ on large amplitude motion by quantum Monte Carlo techniques.

The first experimental observation of the $\mathrm{NH}_{3}-\mathrm{H}_{2}$ complex is due to Moroz et al. ${ }^{4}$ who studied the interaction between several Lewis bases (including ammonia) and molecular hydrogen in an argon matrix. Vibrational spectra have been recorded recently by Jacox and Thompson ${ }^{5}$ in a neon matrix. These authors also performed RMP2 calculations which yielded a coaxial $C_{3 v}$ minimum energy arrangement and electronic dissociation energies of $215 \mathrm{~cm}^{-1}$ and $237 \mathrm{~cm}^{-1}$ at MP2 and $\operatorname{CCSD}(\mathrm{T})$ levels, respectively. In both studies the $\nu_{H H}$ vibration which is dipole forbidden in free $\mathrm{H}_{2}$ was observed. This symmetry breaking is consistent with the colinear $\mathrm{H}_{2}$ orientation in the complex.

The first electronic structure calculation on the $\mathrm{NH}_{5}$ system was undertaken by Olah and coworkers in their search for the hypothetical ammonium hydride. ${ }^{13}$ The potential energy surfaces used in the earlier scattering studies $^{3}$ were based on Hartree-Fock calculations of the repulsive short range part with additional empirical long range dispersion terms and cannot be considered as reliable in the well region. The inversion motion of ammonia was experimentally found to be extremely sensitive to the presence of weakly bound partners like helium. ${ }^{11}$ This observation has motivated the construction of a high quality potential energy surface for the related but more weakly bound $\left(\approx 35 \mathrm{~cm}^{-1}\right) \mathrm{NH}_{3}-\mathrm{He}$ complex. ${ }^{14}$ It may also be noted that the $\mathrm{H}_{2} \mathrm{O}-\mathrm{H}_{2}$ complex has been the subject of high level scattering studies ${ }^{15,16}$ which have inspired the recent construction of a full nine-dimensional potential surface. ${ }^{17}$

The main purpose of our current study of $\mathrm{NH}_{3}-\mathrm{H}_{2}$ was to investigate characteristic features of the potential energy surface in the well region with a high level of accuracy. In addition to the equilibrium structure and the binding energy, we have also studied various angular cuts of the surface which are expected to dominate the internal motion of the complex. This work represents a first step of our project which aims at understanding the internal molecular dynamics of van der Waals complexes, such as $\mathrm{NH}_{3}-\mathrm{H}_{2}$ and $\mathrm{H}_{2} \mathrm{O}-\mathrm{N}_{2}$, by accurate variational methods.

$A b$ initio calculations for the $\mathrm{NH}_{3}-\mathrm{H}_{2}$ complex in 
the electronic ground state have been carried out with coupled cluster techniques. The aug-cc-pVXZ family of basis functions was employed to approach the oneparticle basis-set limit (Section II). The basis set superposition error arising from the supermolecule approach (Section II A) and suitable internal coordinates (Section II B) were considered. Our results (Section III) include first the geometrical and electric properties of the two monomers (Section III A). The internal geometry of the complex has been optimized via numerical gradient techniques (Section III B) and the dissociation energy has been estimated (Section IIIC). The potential energy along the intermolecular distance coordinate (Section IIID) and along several angular coordinates (Section IIIE) have been computed with and without CP correction.

\section{DETAILS OF THE THEORETICAL TREATMENT}

The $a b$ initio computations have been carried out using the coupled-cluster $\operatorname{CCSD}(\mathrm{T})$ method with all single and double substitutions relative to the reference Hartree-Fock determinant, including connected triple excitations in a perturbative fashion. ${ }^{18,19}$ Augmented correlation consistent double-zeta $(\mathrm{X}=\mathrm{D})$, triple-zeta $(\mathrm{X}=\mathrm{T})$, and quadruple-zeta $(\mathrm{X}=\mathrm{Q})$ polarized valence basis sets developed by Dunning et al. ${ }^{20}$ were employed. These sets of basis functions, identified as aug-cc-pVXZ, were constructed by adding diffuse functions to the standard cc-pVXZ basis. In the case of e.g. aug-cc-pVQZ, the diffuse functions $(1 s, 1 p, 1 d, 1 f, 1 g)$ for $\mathrm{N}$ and $(1 s, 1 p, 1 d, 1 f)$ for $\mathrm{H}$ are added to the respective cc-pVQZ sets, leading to $(13 s, 7 p, 4 d, 3 f, 2 g) \rightarrow[6 s, 5 p, 4 d, 3 f, 2 g]$ for nitrogen and $(7 s, 4 p, 3 d, 2 f) \rightarrow[6 s, 5 p, 4 d, 3 f, 2 g]$ for hydrogen. The aug-cc-pVQZ basis set for the $\mathrm{NH}_{3}-\mathrm{H}_{2}$ complex contains 310 contracted Gaussian-type orbitals.

The reference $(\mathrm{SCF})$ electronic configuration of the electronic ground $\tilde{\mathrm{X}}^{1} A_{1}$ state of $\mathrm{NH}_{3}$ is given by $\left(1 a_{1}\right)^{2}\left(2 a_{1}\right)^{2}(1 e)^{4}\left(3 a_{1}\right)^{2}$. Like in water and hydrogen fluoride, the highest occupied orbital of ammonia is the nonbonding orbital $\left(3 a_{1}\right)$, representing a lone electron pair localized on nitrogen.

The quality of coupled cluster results representing a single reference electron correlation strategy may be assessed by means of the $T_{1}$ diagnostic. ${ }^{21}$ In the case of the $\mathrm{NH}_{3}-\mathrm{H}_{2}$ complex, $T_{1}$ values of ca. 0.008 were found, indicating a very low multireference character and confirming the applicability of $\operatorname{CCSD}(\mathrm{T})$ for the present system.

All electronic structure calculations described in this article were performed with the MOLPRO quantum chemistry package. ${ }^{22}$ For each of the basis sets, the structural parameters were optimized by numerical gradient techniques, ${ }^{23}$ as implemented in MOLPRO for the CCSD(T) approach.

\section{A. Computation of interaction energies}

The properties of the $\mathrm{NH}_{3}-\mathrm{H}_{2}$ complex were studied in the supermolecular approach, in which the interaction energy $E_{\text {int }}$ between two monomers A and B is computed as the difference between the supermolecule (complex) and monomer energies,

$$
E_{\text {int }}=E_{A B}(A B)-E_{A}(A)-E_{B}(B) .
$$

In the latter equation, the basis set used to compute $E_{Q}$ for the species $Q$ is shown in parentheses.

In supermolecular electronic structure calculations for weakly bonded complexes the use of finite basis sets may lead to significant effects of the so-called Basis Set Superposition Error (BSSE), introduced by a different number of basis functions included in the complex and monomer calculations. BSSE usually leads to an overestimation of the interaction.

The interaction energies of Eq. (1) were corrected for BSSE by means of the counterpoise (CP) correction proposed by Boys and Bernardi. ${ }^{24}$ The $\mathrm{CP}$ corrected results $E_{i n t}^{\mathrm{CP}}$ for the interaction energy were obtained by evaluating the monomer wave functions in the complex centered basis set as

$$
E_{\text {int }}^{\mathrm{CP}}=E_{A B}(A B)-E_{A}(A B)-E_{B}(A B) .
$$

All complex geometries were fully optimized at the $\mathrm{CP}$ uncorrected level, such that the interaction energies of Eq. (2) are actually a posteriori corrected results.

The CP corrected equilibrium structure may, however, differ from the corresponding $\mathrm{CP}$ uncorrected value. In this fashion, the $\mathrm{CP}$ methodology may in principle change the topography of the overall potential energy surface. We report both $\mathrm{CP}$ corrected and uncorrected cuts through the $\mathrm{NH}_{3}-\mathrm{H}_{2}$ surface with basis sets of systematically increasing quality in order to assess the reliability and convergence of this procedure and the robustness of physically relevant features of the potential energy surface. ${ }^{25,26}$

\section{B. Coordinates}

For floppy complexes a choice of coordinates which allow the easy exploitation or verification of existing symmetries is of great importance. The geometrical arrangements of the $\mathrm{NH}_{3}-\mathrm{H}_{2}$ complex are described in terms of the four internal vectors shown in Fig. 1. The bond distance vector $\mathbf{d}$ (length $d$ ) for the $\mathrm{H}_{2}$ part is used in combination with the trisector vector ${ }^{(3)} \mathbf{s}$ (length $d_{\mathrm{N}}$ ) and the $\mathrm{H}_{3}$ plane vector $\mathbf{p}$ (length $d_{\mathrm{H}}$ ) for the $\mathrm{NH}_{3}$ part. The trisector denotes a spatial direction which encloses the same angle with each of the $\mathrm{N}-\mathrm{H}$ bond-distance vectors of $\mathrm{NH}_{3}$. For an explicit definition of ${ }^{(3)} \mathbf{s}$ see Ref. 27. The vector $\mathbf{p}$ connects the $\mathrm{H}_{3}$ center of mass to one of the three $\mathrm{H}$ atoms of ammonia. The vector $\mathbf{R}$ joins the center of mass of the $\mathrm{H}_{2}$ molecule with the $\mathrm{N}$ atom. 


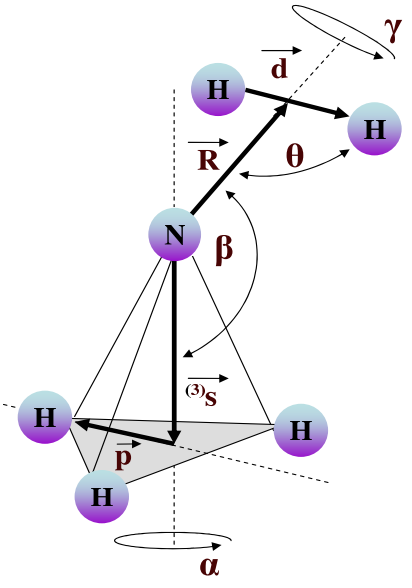

FIG. 1: Description of the internal geometry of the intermolecular complex $\mathrm{NH}_{3}-\mathrm{H}_{2}$ with pyramidal $\mathrm{C}_{3 v}$ ammonia. The lengths of the four vectors $\mathbf{d}, \mathbf{R},{ }^{(3)} \mathbf{s}$, and $\mathbf{p}$ are $d, R, d_{\mathrm{N}}$, and $d_{\mathrm{H}}$, respectively. The four angular coordinates $\{\alpha, \beta, \gamma, \theta\}$ describe a mutual orientation of the four internal vectors. For more details, see the text.

TABLE I: Transformation of the angular coordinates $\alpha, \beta, \gamma$, and $\theta$ under the spatial inversion $\hat{\mathcal{J}}_{v}$, the exchange $\hat{\sigma}_{3}\left(\mathrm{NH}_{3}\right)$ of the three $\mathrm{H}$ atoms in $\mathrm{NH}_{3}$, and the exchange $\hat{\sigma}_{2}\left(\mathbf{H}_{2}\right)$ of the $\mathrm{H}$ atoms in $\mathrm{H}_{2}$.

\begin{tabular}{cccc}
\hline \hline & $\hat{\mathcal{J}}_{v}$ & $\hat{\sigma}_{3}\left(\mathrm{NH}_{3}\right)$ & $\hat{\sigma}_{2}\left(\mathbf{H}_{2}\right)$ \\
\hline & & & \\
$\alpha$ & $2 \pi-\alpha$ & $\alpha+k \pi / 3(k=0,1,2)$ & $\alpha$ \\
$\beta$ & $\beta$ & $\beta$ & $\beta$ \\
$\gamma$ & $2 \pi-\gamma$ & $\gamma$ & $\pi+\gamma$ \\
$\theta$ & $\theta$ & $\theta$ & $\pi-\theta$ \\
\hline \hline
\end{tabular}

For ammonia possessing the pyramidal $\mathrm{C}_{3 v}$ structure, when $\left(\mathbf{p},{ }^{(\mathbf{3})} \mathbf{s}\right)=\mathbf{0}$ holds, the coordinate space of Fig. 1 can be parametrized in terms of eight coordinates: four lengths, $d, d_{\mathrm{N}}, d_{\mathrm{H}}$, and $R$, two polar angles, $\theta$ and $\beta$, and two dihedral angles, $\alpha$ and $\gamma$, where $\beta, \theta \in(0, \pi)$ and $\alpha, \gamma \in(0,2 \pi)$. The angles $\beta$ and $\alpha$ represent the polar and azimuthal angle of $\mathbf{R}$ in the $z \wedge x$ frame given by ${ }^{(3)} \mathbf{s} \wedge$ $\mathbf{p}$, whereas the angles $\theta$ and $\gamma$ specify the orientation of the $\mathrm{H}_{2}$ vector $\mathbf{d}$ in the frame $\mathbf{R} \wedge{ }^{(3)} \mathbf{s}$. For rigid monomers, the three distances $d, d_{\mathrm{N}}$, and $d_{\mathrm{H}}$ assume constant values, such that the geometry of the complex is described by five internal coordinates, namely the intermolecular distance $R$ and four angular coordinates $\theta, \alpha, \beta, \gamma$.

The internal coordinates $\left\{d, d_{\mathrm{N}}, d_{\mathrm{H}}, R, \alpha, \beta, \gamma, \theta\right\}$ of Fig. 1 were used to construct the $\mathrm{Z}$ matrices for the electronic structure calculations. The symmetry properties of the angular coordinates $\alpha, \beta, \gamma, \theta$ with respect to the spatial inversion $\hat{\mathcal{J}}_{v}$, the permutation (threefold rotational) symmetry $\hat{\sigma}_{3}\left(\mathrm{NH}_{3}\right)$ of $\mathrm{NH}_{3}$, and the permutation (exchange) symmetry $\hat{\sigma}_{2}\left(\mathrm{H}_{2}\right)$ of the $\mathrm{H}_{2}$ molecules are summarized in Table I. The four distance coordinates $d, d_{\mathrm{N}}, d_{\mathrm{H}}, R$ remain unaltered under the action of $\hat{\mathcal{J}}_{v}$,
$\hat{\sigma}_{3}\left(\mathrm{NH}_{3}\right)$, and $\hat{\sigma}_{2}\left(\mathrm{H}_{2}\right)$. Owing to the specific symmetries shown in Table I, the angular space $\{\alpha, \beta, \gamma, \theta\}$ of $\mathrm{NH}_{3^{-}}$ $\mathrm{H}_{2}$ may readily be reduced to $\alpha \in(0, \pi / 3), \beta \in(0, \pi)$, $\gamma \in(0, \pi)$, and $\theta \in(0, \pi / 2)$ in the actual ab initio computation. For given $\beta$ and $\left\{d, d_{\mathrm{N}}, d_{\mathrm{H}}, R\right\}$, twelve angular points specified by $(\alpha, \gamma, \theta),(\alpha+2 \pi / 3, \gamma, \theta)$, $(\alpha+4 \pi / 3, \gamma, \theta),(2 \pi-\alpha, 2 \pi-\gamma, \theta),(2 \pi / 3-\alpha, 2 \pi-\gamma, \theta)$, $(4 \pi / 3-\alpha, 2 \pi-\gamma, \theta),(\alpha, \gamma+\pi, \pi-\theta),(\alpha+2 \pi / 3, \gamma+$ $\pi, \pi-\theta),(\alpha+4 \pi / 3, \gamma+\pi, \pi-\theta),(2 \pi-\alpha, \pi-\gamma, \pi-\theta)$, $(2 \pi / 3-\alpha, \pi-\gamma, \pi-\theta),(4 \pi / 3-\alpha, \pi-\gamma, \pi-\theta)$ possess the same potential energy.

In the following text, we also make use of the $\mathrm{H}-\mathrm{N}-\mathrm{H}$ angle $\delta$ and the umbrella coordinate $\rho$. The latter two angles, $\delta$ and $\rho$, for pyramidal $C_{3 v}$ ammonia are related to the distance $d_{\mathrm{H}}$ shown in Fig. 1 by the expressions $d_{\mathrm{H}}=r_{\mathrm{NH}} \sin \rho$ and $(\sqrt{3} / 2) d_{\mathrm{H}}=r_{\mathrm{NH}} \sin (\delta / 2)$.

\section{RESULTS}

\section{A. Monomer properties}

Electrostatic and induction forces make major contributions to the $\mathrm{NH}_{3}-\mathrm{H}_{2}$ interaction and are responsible for long-range attraction between the two monomers. In the first step of our study, we have thus examined, in addition to the geometrical parameters of the monomers, also their electric properties in order to verify that an appropriate theoretical approach was being employed. At the $\operatorname{CCSD}(\mathrm{T})$ level of theory, the electric dipole moments $\mu_{q}$, electric dipole polarizabilities $\alpha_{q_{1} q_{2}}$, and electric quadrupole moments $\Theta_{q_{1} q_{2}}$ for $q=x, y, z$ were obtained numerically by means of finite field calculations, using field strengths of 0.005, 0.0025, and 0.001 a.u. Throughout this paper, we use atomic units for the electric properties, i.e. $e a_{0}$ for the dipole moment, $a_{0}^{3}$ for the polarizability, and $e a_{0}^{2}$ for the quadrupole moment.

Our results for ammonia and molecular hydrogen are reported in Table II, along with available experimental data. For the mean polarizability $\bar{\alpha}$ and anisotropy $\Delta \alpha$, we use the common definitions ${ }^{33}$

$$
\begin{aligned}
\bar{\alpha} & =\left(\alpha_{x x}+\alpha_{y y}+\alpha_{z z}\right) / 3, \\
\Delta \alpha & =\alpha_{z z}-\left(\alpha_{x x}+\alpha_{y y}\right) / 2 .
\end{aligned}
$$

Since the quadrupole moment $\Theta$ is given by a traceless second moment tensor, $\Theta_{x x}+\Theta_{y y}=-\Theta_{z z}$, such that $\Theta_{x x}=\Theta_{y y}=-\Theta_{z z} / 2$ for the systems studied here.

The MP2 values listed in Table II for $\mu_{z}, \alpha$, and $\Theta_{z z}$ were computed for the equilibrium configurations optimized at the CCSD(T) level. In view of our future work, it was of particular interest to test which level of agreement between MP2 and CCSD(T) electric properties can be expected for $\mathrm{NH}_{3}-\mathrm{H}_{2}$. Due to the significantly lower computational effort for MP2, a MP2 dipole moment surface is often used in combination with a $\operatorname{CCSD}(\mathrm{T})$ potential energy surface in molecular rovibrational studies. ${ }^{34}$ The reliability of the electric property values computed 
TABLE II: Equilibrium geometry and electric properties of the $\mathrm{H}_{2}$ and $\mathrm{NH}_{3}$ monomers from CCSD(T) calculations. The MP2 results for $\mu_{z}, \bar{\alpha}, \Delta \alpha$, and $\Theta_{z z}$ were evaluated at the optimized $\operatorname{CCSD}(\mathrm{T})$ geometry. The bond distances are in bohr, bond angles in degrees, and electric properties in atomic units. The superscript given along with the experimental values refers to the source reference.

\begin{tabular}{|c|c|c|c|c|c|c|c|c|c|}
\hline & \multicolumn{3}{|r|}{$\mathrm{H}_{2}$} & \multirow[b]{2}{*}{$\mathrm{r}_{e}(\mathrm{NH})$} & \multirow[b]{2}{*}{$\angle(\mathrm{HNH})_{e}$} & \multicolumn{3}{|c|}{$\mathrm{NH}_{3}$} & \multirow[b]{2}{*}{$\Theta_{z z}$} \\
\hline & $\mathrm{r}_{e}(\mathrm{HH})$ & $(\bar{\alpha}, \Delta \alpha)$ & $\Theta_{z z}$ & & & $\rho_{e}$ & $\mu_{z}$ & $(\bar{\alpha}, \Delta \alpha)$ & \\
\hline $\begin{array}{c}\text { aug-cc-pVTZ } \\
\text { MP2 }\end{array}$ & 1.404 & $\begin{array}{l}(5.22,1.81) \\
(5.23,1.83)\end{array}$ & $\begin{array}{l}0.463 \\
0.478\end{array}$ & 1.918 & 106.4 & 112.4 & $\begin{array}{l}0.598 \\
0.604\end{array}$ & $\begin{array}{l}(14.08,1.50) \\
(14.20,1.69)\end{array}$ & $\begin{array}{l}-2.168 \\
-2.216\end{array}$ \\
\hline $\begin{array}{c}\text { aug-cc-pVQZ } \\
\text { MP2 }\end{array}$ & 1.402 & $\begin{array}{l}(5.20,1.82) \\
(5.21,1.84)\end{array}$ & $\begin{array}{l}0.458 \\
0.474\end{array}$ & 1.914 & 106.6 & 112.2 & $\begin{array}{l}0.599 \\
0.604\end{array}$ & $\begin{array}{l}(14.06,1.58) \\
(14.19,1.82)\end{array}$ & $\begin{array}{l}-2.178 \\
-2.225\end{array}$ \\
\hline $\exp$ & $1.401^{28}$ & $5.314^{29}$ & $0.460 \pm 0.021^{30}$ & $1.913^{28}$ & $106.67^{28}$ & $112.15^{28}$ & $0.594^{31}$ & $14.19^{29}$ & $-2.45 \pm 0.3^{32}$ \\
\hline
\end{tabular}

by finite differencing, the only approach available at the CCSD(T) level, was verified at the MP2 level by comparing finite-field results with dipole moment expectation values computed by means of analytical energy derivatives available for MP2 in the MOLPRO implementation.

Our results for $\mathrm{H}_{2}$ and $\mathrm{NH}_{3}$ at the $\operatorname{CCSD}(\mathrm{T})$ and MP2 levels are generally in very good agreement with the available experimental data ${ }^{28-30,32}$ and other theoretical results. ${ }^{31,35-37}$ The experimental molecular polarizabilities $\bar{\alpha}$ listed in Table II for $\mathrm{H}_{2}$ and $\mathrm{NH}_{3}$ were obtained from dipole (e,e) energy-loss spectra. ${ }^{29}$ The experimental quadrupole $\Theta_{z z}$ moment of $\mathrm{H}_{2}$ was derived ${ }^{30}$ from the molecular susceptibilities known from molecular beam experiments. ${ }^{38}$ For both monomers, experimental ground-state rovibrational quadrupole moments are reported in Table II. One may, however, note that the dipole moment provided for $\mathrm{NH}_{3}$ corresponds to a vibrationless situation. ${ }^{31}$ For a detailed theoretical study of the equilibrium dipole moment of ammonia, see the paper by Halkier and Taylor. ${ }^{37}$

The molecular parameters of Table II computed at the $\operatorname{CCSD}(\mathrm{T}) /$ aug-cc-pVQZ level agree within $4 \%$ with the corresponding experimental results, with the exception of $\Theta_{z z}$ for $\mathrm{NH}_{3}$. The discrepancy of ca. 11\%, found in this case, is probably due to a prominent vibrational correction to the equilibrium quadrupole moment. A vibrational correction of about $-0.13 e a_{0}^{2}$ was estimated by Piecuch et al. ${ }^{31}$ in their study of the quadrupole moment of ammonia. This would account for about half of our discrepancy.

For molecular hydrogen, our CCSD(T)/aug-cc-pVQZ values for the mean dipole polarizability of $5.20 e a_{0}$ and the anisotropy of $1.82 e a_{0}$ agree very well with the results of Kołos and Wolniewicz, ${ }^{35}$ who calculated $\bar{\alpha}$ and $\Delta \alpha$ of 5.179 and $1.803 a_{0}^{3}$, respectively, for an internuclear separation of $1.40 a_{0}$. For the ground vibrational state of $\mathrm{H}_{2}$, Kołos and Wolniewicz obtained a value of 5.414 and $2.024 a_{0}^{3}$, respectively. Our results for $\bar{\alpha}$ and $\Delta \alpha$ of ammonia are in very good agreement with the values of 14.01 and $1.52 a_{0}$ computed recently by Lim et al. ${ }^{36}$ at the $\operatorname{CCSD}(\mathrm{T}) /$ aug-cc-pVTZ level.

\section{B. Equilibrium structure of the complex}

The equilibrium structure of the $\mathrm{NH}_{3}-\mathrm{H}_{2}$ complex is found to be axial, with molecular hydrogen colinear with the $C_{3}$ symmetry axis of ammonia. The $\mathrm{H}_{2}$ monomer approaches $\mathrm{NH}_{3}$ from the nitrogen side. This axial arrangement is described by $\beta_{e}=\pi$ and $\theta_{e}=0, \pi$ in terms of the coordinates shown in Fig. 1. The other structural parameters, obtained at the $\operatorname{CCSD}(\mathrm{T})$ level of theory with the aug-cc-pVXZ basis sets $(\mathrm{X}=\mathrm{D}, \mathrm{T}, \mathrm{Q})$ are gathered in Table III. One may note that the geomery optimization is carried out at the CP uncorrected level. For the augcc-pVXZ series, the convergence of the cc-pVQZ results appears to be better than $0.005 \mathrm{a}_{0}$ for the bond lengths and $0.1^{\circ}$ for the angular coordinates.

An axial equilibrium structure for $\mathrm{NH}_{3}-\mathrm{H}_{2}$ was also found in the previous study by Jacox and Thompson $^{5}$, who obtained an equilibrium configuration $\left[d_{e}, r_{e}(\mathrm{NH}), \mathrm{R}_{\mathrm{e}}, \delta_{\mathrm{e}}, \rho_{\mathrm{e}}\right]$ given at the $\mathrm{RMP} 2 / 6$ $311++\mathrm{G}(3 \mathrm{df}, 3 \mathrm{pd})$ level of theory by $\left[1.397 \mathrm{a}_{0}, 1.912 \mathrm{a}_{0}\right.$, $5.469 \mathrm{a}_{0}, 106.7^{\circ}, 111.9^{\circ}$ ]. In Ref. 5 , the electronic dissociation energy is estimated to be about $215 \mathrm{~cm}^{-1}$ at the MP2 level.

The monomer coordinates change only slightly upon complexation, as seen by a comparison of Tables II and III. Whereas the ammonia moiety exhibits variations within only about $0.001 \mathrm{a}_{0}$ and $0.1^{\circ}$, the bond length of hydrogen increases by ca. $0.005 \mathrm{a}_{0}$ in the complex. The effect of the fragment relaxation to the electronic binding energy is found to be small and on the order of $1 \mathrm{~cm}^{-1}$, again with a markedly asymmetric distribution over the 
TABLE III: Structural parameters of the intermolecular complex $\mathrm{NH}_{3}-\mathrm{H}_{2}$ obtained at the $\operatorname{CCSD}(\mathrm{T}) /$ aug-cc-pVXZ level $(\mathrm{X}=\mathrm{D}, \mathrm{T}, \mathrm{Q})$. The MP2 and CCSD results shown here are computed for the $\operatorname{CCSD}(\mathrm{T})$ optimized geometries. The superscript $\mathrm{CP}$ indicates counterpoise corrected results. The bond distances are given in bohr, angles in degrees, dissociation energies in $\mathrm{cm}^{-1}$, and electric properies in atomic units. The angle $\delta$ stands for $\angle(\mathrm{HNH})$ and $\rho$ for the umbrella angle. For the definition of the other coordinates, see the text.

\begin{tabular}{lccc}
\hline \hline & aug-cc-pVDZ & aug-cc-pVTZ & aug-cc-pVQZ \\
\hline$d_{e}$ & 1.444 & 1.408 & 1.406 \\
$d_{\mathrm{N}, \mathrm{e}}$ & 0.750 & 0.731 & 0.726 \\
$d_{\mathrm{H}, \mathrm{e}}$ & 1.783 & 1.773 & 1.771 \\
$r_{e}(\mathrm{NH})$ & 1.934 & 1.918 & 1.914 \\
$R_{e}$ & 5.915 & 6.010 & 6.010 \\
$\delta_{e}$ & 105.9 & 106.4 & 106.5 \\
$\rho_{e}$ & 112.8 & 112.4 & 112.3 \\
$D_{e}$ & & & \\
$D_{e}^{\mathrm{CP}}$ & 326.4 & 271.0 & 260.0 \\
$D_{e}^{\mathrm{CP}}(\mathrm{MP} 2)$ & 201.3 & 242.1 & 249.6 \\
$D_{e}^{\mathrm{CP}}(\mathrm{CCSD})$ & 196.1 & 229.1 & 237.1 \\
& 177.9 & 216.5 & 222.7 \\
$\mu_{z}$ & & & \\
$\mu_{z}(\mathrm{MP} 2)$ & 0.686 & 0.672 & 0.672 \\
$\bar{\alpha}$ & 0.694 & 0.678 & 0.677 \\
$\bar{\alpha}(\mathrm{MP} 2)$ & 19.60 & 19.50 & 19.43 \\
$\Delta \alpha$ & 19.67 & 19.63 & 19.58 \\
$\Delta \alpha(\mathrm{MP} 2)$ & 6.18 & 5.45 & 5.44 \\
$\Theta_{z z}$ & 6.22 & 5.61 & 5.64 \\
$\Theta_{z z}(\mathrm{MP} 2)$ & -1.699 & -1.578 & -1.583 \\
\hline \hline & -1.726 & -1.609 & -1.615 \\
\hline \hline
\end{tabular}

monomers amounting to contributions of ca. $0.01 \mathrm{~cm}^{-1}$ and $0.90 \mathrm{~cm}^{-1}$ for $\mathrm{NH}_{3}$ and $\mathrm{H}_{2}$, respectively.

The equilibrium rotational constants for the complex $\mathrm{NH}_{3}-\mathrm{H}_{2}$ and for the two monomers, $\mathrm{H}_{2}$ and $\mathrm{NH}_{3}$, are summarized here for the equilibrium CCSD(T)/aug-ccpVQZ geometry of Tables II and III. For the complex, the values of $A_{e}, B_{e}, C_{e}$ are calculated to be 6.348 , $0.678,0.678 \mathrm{~cm}^{-1}$ (prolate symmetric top). For ammonia, we obtain 9.951, 9.951, $6.346 \mathrm{~cm}^{-1}$ (oblate symmetric top), which may be compared with the experimental $A_{0}, B_{0}, C_{0}$ results $^{28}$ of $9.9443,9.9443,6.196 \mathrm{~cm}^{-1}$. For molecular hydrogen, the computed value for $B_{e}$ of 60.76 $\mathrm{cm}^{-1}$ compares very well with the value extracted from experiments $^{39} B_{e}=60.853 \mathrm{~cm}^{-1}$.

The harmonic frequencies of the $\mathrm{NH}_{3}-\mathrm{H}_{2}$ complex, computed by numerical second derivatives at the equilibrium geometry of TableIII, are compared with the harmonic frequencies of the monomers in Table IV. The frequencies near $4400 \mathrm{~cm}^{-1}$ and $1060 \mathrm{~cm}^{-1}$ corresponding to the $\mathrm{H}-\mathrm{H}$ stretching mode of the $\mathrm{H}_{2}$ subunit and to the inversion of the $\mathrm{NH}_{3}$ moiety exhibit changes of ca. $-38 \mathrm{~cm}^{-1}$ and $+7 \mathrm{~cm}^{-1}$, respectively, upon complexation. In the complex, there are five low frequency modes, associated with intermolecular motions. In a system as
TABLE IV: Harmonic frequencies (in $\mathrm{cm}^{-1}$ ) of the $\mathrm{NH}_{3}-\mathrm{H}_{2}$ complex and the monomers, $\mathrm{H}_{2}$ and $\mathrm{NH}_{3}$, calculated for the equilibrium geometries of Table I and Table III, employing the CCSD(T) method in combination with the aug-cc-pVTZ and aug-cc-pVQZ basis sets.

\begin{tabular}{lccccc}
\hline \hline & \multicolumn{2}{c}{ aug-cc-pVTZ } & & \multicolumn{2}{c}{ aug-cc-pVQZ } \\
\cline { 2 - 3 } \cline { 5 - 6 } & $\mathrm{NH}_{3}-\mathrm{H}_{2}$ & $\mathrm{H}_{2} / \mathrm{NH}_{3}$ & & $\mathrm{NH}_{3}-\mathrm{H}_{2}$ & $\mathrm{H}_{2} / \mathrm{NH}_{3}$ \\
\hline$\omega_{1}(\mathrm{H}-\mathrm{H})$ & 4363 & 4401 & & 4361 & 4399 \\
$\omega_{2,3}$ & 3591 & 3592 & & 3605 & 3607 \\
$\omega_{4}$ & 3462 & 3464 & & 3475 & 3476 \\
$\omega_{5,6}$ & 1672 & 1672 & & 1674 & 1674 \\
$\omega_{7}$ & 1070 & 1063 & & 1066 & 1059 \\
$\omega_{8,9}$ & 344 & & & 329 & \\
$\omega_{10}$ & 141 & & & 139 & \\
$\omega_{11}$ & 48 & & & 42 & \\
$\omega_{12}$ & 46 & & 31 & \\
\hline \hline
\end{tabular}

anharmonic and floppy as $\mathrm{NH}_{3}-\mathrm{H}_{2}$ harmonic frequencies can only be very crude approximations. Besides clearly identifying the structure as a true minimum they show, however, interesting trends: The amount of the hydrogen vibrational red shift significantly exceeds the shift of any of the ammonia vibrational modes. The ammonia inversion motion shows a noticeable blue shift, as experimentally observed for $\mathrm{NH}_{3}$ embedded into liquid helium clusters $^{11}$.

In the present study, only valence correlations were evaluated. The importance of core correlation was tested for the aug-cc-pCVTZ basis set. ${ }^{40}$ We found that the $\operatorname{CCSD}(\mathrm{T}) / \mathrm{aug}$-cc-pCVTZ equilibrium structure $\left[d_{e}, r_{e}(\mathrm{NH}), \mathrm{R}_{\mathrm{e}}, \rho_{\mathrm{e}}\right]$ given by $\left[1.409 a_{0}, 1.916 a_{0}, 6.007 a_{0}\right.$, $\left.112.6^{\circ}\right]$ for the all electron correlation treatment shows excellent agreement with the frozen-core $\operatorname{CCSD}(\mathrm{T}) /$ augcc-pVTZ geometry of Table III. The binding energy at the aug-cc-pCVTZ level was found to be 270.7 and 242.9 $\mathrm{cm}^{-1}$ in the uncorrected and counterpoise corrected situation, respectively, deviating by 0.3 and $0.8 \mathrm{~cm}^{-1}$ from the corresponding values of Table III.

\section{Dissociation energy}

The classical binding (dissociation) energies in Table III were computed at equilibrium as $D_{e}=-E_{\text {int }}$ and $D_{e}^{\mathrm{CP}}=-E_{\text {int }}^{\mathrm{CP}}$ with the help of Eqs. (1) and (2). Note that the parameters with the superscript $\mathrm{CP}$ refer to counterpoise corrected results. The values for $D_{e}$ and $D_{e}^{\mathrm{CP}}$ at second order Møller-Plesset perturbation theory (MP2) level and coupled cluster CCSD level were estimated for the optimized $\operatorname{CCSD}(\mathrm{T})$ geometries. The MP2 and CCSD results for $D_{e}^{\mathrm{CP}}$ in Table III are lower by up to $13 \mathrm{~cm}^{-1}(5 \%)$ and $27 \mathrm{~cm}^{-1}(11 \%)$, respectively, than the corresponding $\operatorname{CCSD}(\mathrm{T})$ values.

Since BSSE introduces a nonphysical attraction be- 


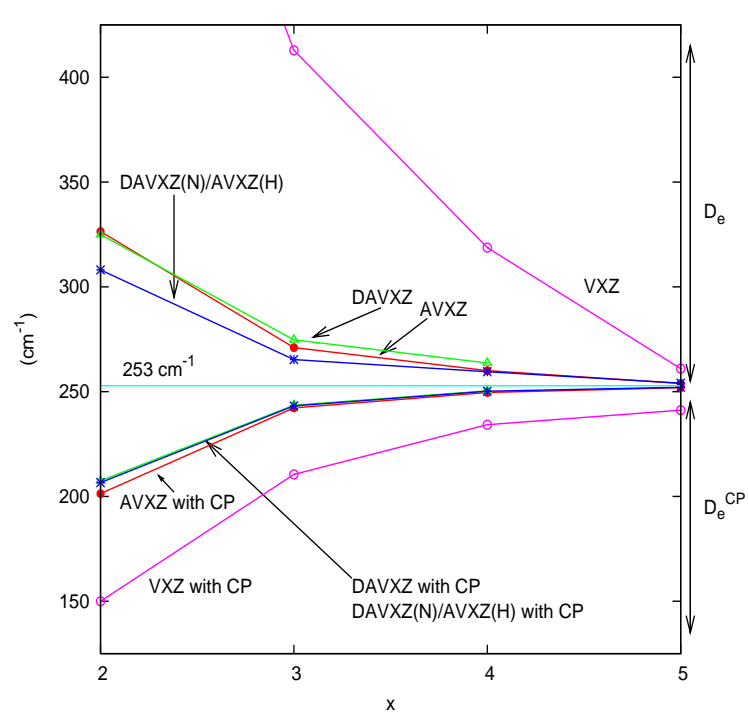

FIG. 2: Variation of the uncorrected $D_{e}$ and counterpoise corrected $D_{e}^{\mathrm{CP}}$ dissociation energy with the basis set cardinal number $x=2-5$, where $x=2,3,4$ stands for $\mathrm{X}=\mathrm{D}, \mathrm{T}, \mathrm{Q}$, respectively. The basis sets cc-pVXZ, aug-cc-pVXZ, and d-augcc-pVXZ are denoted by VXZ, AVXZ, and DAVXZ, respectively. The basis set employing the d-aug-cc-pVXZ functions for nitrogen and aug-cc-pVXZ for hydrogen is abbreviated as $\operatorname{DAVXZ}(\mathrm{N}) / \operatorname{AVXZ}(\mathrm{H})$.

tween monomers, CP corrections lead to a less stable complex and $D_{e}^{\mathrm{CP}}$ values smaller than the corresponding $D_{e}$. For the $\mathrm{NH}_{3}-\mathrm{H}_{2}$ complex in Table III, $D_{e}^{\mathrm{CP}}$ is lower by ca. $125,29,10 \mathrm{~cm}^{-1}$ than $D_{e}$ for the aug-ccpVXZ series with $\mathrm{X}=\mathrm{D}, \mathrm{T}, \mathrm{Q}$, respectively. As expected, the residual BSSE effect is larger for the smallest basis set. While both series of $D_{e}$ values appear to converge in a satisfactory way towards a common limit it is evident that the standard CP correction is overshooting noticeably and that corrected values are only slightly better than uncorrected ones, an effect that has been reported previously for other intermolecular complexes ${ }^{25}$.

As an attempt to assess the complete basis set limit, the dissociation energies of Table III were approximated in a simple fashion by

$$
D_{e}(x)=D_{e}^{\infty}+A e^{-b x},
$$

where $x=2,3,4$ for the basis set cardinal number $\mathrm{X}=\mathrm{D}, \mathrm{T}, \mathrm{Q}$. This extrapolation procedure yields $D_{e}^{\infty}$ values of 257 and $251 \mathrm{~cm}^{-1}$ for the uncorrected and CP corrected energies, respectively. Employing an exponential fit to SCF energies and the two-parameter formula $a+b / x^{3}$ for correlation contributions, as previously suggested by Helgaker et al., ${ }^{41}$ we find values of $254 \mathrm{~cm}^{-1}$ and $252 \mathrm{~cm}^{-1}$ from uncorrected and CP corrected energies, respectively. At this step of our study, we carried out also a single point calculation with the aug-cc-pV5Z basis, using the optimized aug-cc-pVQZ geometry of Table III. These calculations have given $D_{e}$ of $254 \mathrm{~cm}^{-1}$ and $D_{e}^{\mathrm{CP}}$ of $252 \mathrm{~cm}^{-1}$ for the aug-cc-pV5Z set, leading to an estimate of ca. $253 \mathrm{~cm}^{-1}$ for the classical binding energy of $\mathrm{NH}_{3}-\mathrm{H}_{2}$.

In order to further investigate the basis set saturation effect, the standard correlation consistent, cc-pVXZ, and doubly augmented correlation consistent, d-aug-ccpVXZ, series were additionally tested for the determination of the dissociation energy of the complex. These results are displayed in Fig. 2. As seen there, the uncorrected $D_{e}$ dissociation energies clearly approach the complete basis set limit from above, whereas the CP corrected $D_{e}^{\mathrm{CP}}$ results do so from below. The cc-pVXZ family approaches the complete basis set limit in the slowest fashion, due to the lack of diffuse functions.

The CP uncorrected results in Fig. 2 for the the daug-cc-pVXZ family are in fact somewhat worse than the corresponding aug-cc-pVXZ values. Furthermore we encountered a linear dependence in this basis set at the relevant geometries. Since the latter effect was found to be due to the d-aug-cc-pV5Z basis sets on the hydrogen atoms of the $\mathrm{H}_{2}$ unit, subsequent calculations were carried out employing a set constructed from the d-augcc-pVXZ functions for nitrogen and aug-cc-pVXZ functions for hydrogen. This basis set is denoted here by $\mathrm{d}-$ aug-cc-pVXZ(N)/aug-cc-pVXZ(H). The performance of the aug-cc-pVXZ family and the combined d-aug-cc$\mathrm{pVXZ}(\mathrm{N}) /$ aug-cc-pVXZ $(\mathrm{H})$ series for $X>2$ appears to a large extent comparable.

The actual $D_{0}$ dissociation energy is related to the electronic dissociation energy of about $250 \mathrm{~cm}^{-1}$ in a non trivial way. The five intermolecular vibrational modes disappear upon dissociation and correlate with fragment translation and rotation. A crude estimate of their zero point energy contribution based on the harmonic frequencies from Table IV would in fact predict the absence of any bound state assuming dissociation into $J=0$ fragments. Massive anharmonic corrections to this estimate are required to allow $D_{0}>0 . D_{0}$ is probably very small and angular momentum constraints could lead to the existence of excited states which are more strongly bound with respect to their rotational asymptote. ${ }^{4,6,7}$

\section{Radial profiles}

The variation of the CCSD $(\mathrm{T}) /$ aug-cc-pVXZ potential energy along the intermolecular distance $R$ is displayed in Fig. 3. The energy profiles shown there have been computed for the axial equilibrium arrangement described by $\beta_{e}=\pi$, assuming rigid monomers at their optimized stucture of Table II. On the right hand side of Fig. 3, we also show the variation of the $z$ component of the dipole moment, $\mu_{z}$, computed at the MP2 level along the distance $R$.

The radial potential energy profiles in Fig. 3 show that the CP correction increases the optimum intermolecular distances. From polynomial fits to calculated radial data near the minimum of Fig. 3 for the aug-cc-pVXZ families of $\mathrm{X}=\mathrm{D}, \mathrm{T}, \mathrm{Q}$, we have determined $R_{\text {opt }}$ of $5.917,6.013$, 

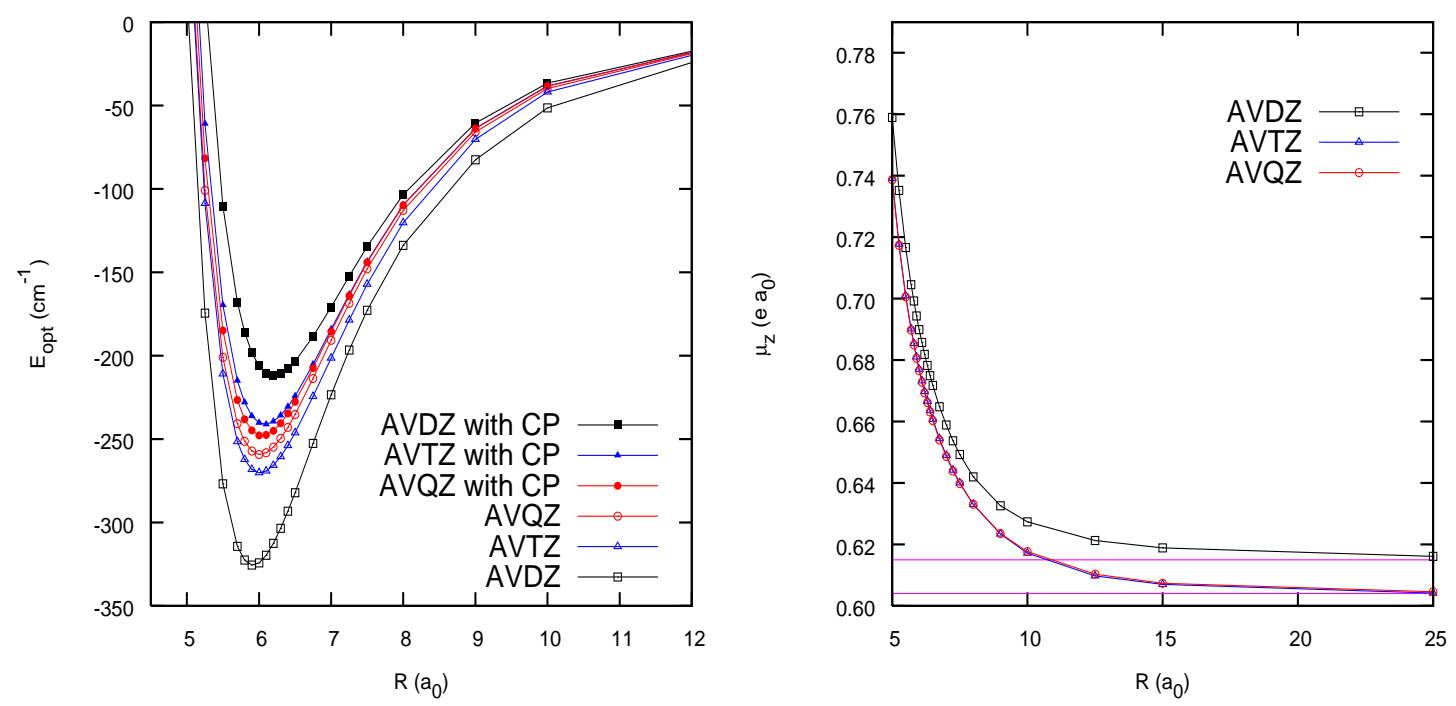

FIG. 3: Variation of the potential energy (left) and variation of the MP2 dipole moment (right) with the intermolecular distance $R$ for the axial equilibrium arrangement of the $\mathrm{NH}_{3}-\mathrm{H}_{2}$ complex. The dipole moment of the free ammonia molecule is indicated by the horizontal lines in the figure on the right hand side. The aug-cc-pVTZ and aug-cc-pVQZ results for $\mu_{z}$ appear identical on the scale of the figure.

$6.013 \mathrm{a}_{0}$ for the uncorrected values and $6.201,6.082$, $6.043 \mathrm{a}_{0}$ for the CP corrected results, respectively. An exponential extrapolation of the $R_{\text {opt }}$ values in the spirit of Eq. 5 gives $R_{o p t}^{\infty}$ of $6.013 \mathrm{a}_{0}$ and $6.025 \mathrm{a}_{0}$ for the uncorrected and $\mathrm{CP}$ corrected situation, respectively. One may note that the inclusion of monomer relaxation in the optimization procedure affects the optimum $R$ by $0.002-$ $0.003 \mathrm{a}_{0}$, as a comparison of the uncorrected $R_{o p t}$ estimated for the data of Fig. 3 and the results of Table III shows.

Compared to the $\mu_{z}$ value of ammonia, the dipole moment $\mu_{z}$ of the $\mathrm{NH}_{3}-\mathrm{H}_{2}$ complex is significantly (ca. $11 \%$ ) enhanced, see Tables II and III. Upon dissociation it slowly approaches the free ammonia limit in Fig. 3 (right) from above. For the aug-cc-pVDZ and augcc-pVQZ basis sets, $\mu_{z}$ assumes values of 0.6189 and $0.6074 e a_{0}^{2}$ at $R=15 \mathrm{a}_{0}$ and of 0.6155 and $0.6046 e a_{0}^{2}$ at $R=25 \mathrm{a}_{0}$, thus exhibiting a lowering of ca. 0.0030 $e a_{0}^{2}$ in this distance range. The $\operatorname{CCSD}(\mathrm{T}) /$ aug-cc-pVQZ and MP2/aug-cc-pVQZ results for $\mu_{z}$ of the free ammonia molecule are 0.599 and $0.604 e a_{0}$, as listed in Table II.

\section{E. Angular profiles}

The intermolecular angular motion plays a major role in the internal dynamics of weakly bound systems, primarily due to its large amplitude character. The latter feature may lead to pronounced coupling between intermolecular angular and radial vibrational modes, as well as to important coupling with the overall rotational momentum. We have thus investigated in some detail the angular properties of the potential energy surface for

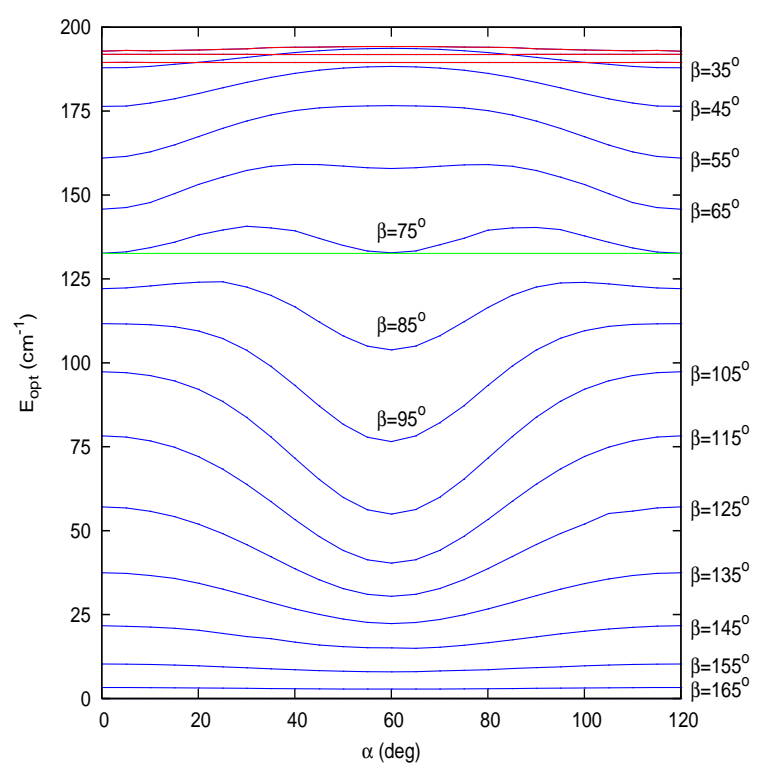

FIG. 4: Variation of the $\operatorname{CCSD}(\mathrm{T}) /$ aug-cc-pVTZ potential energy along the azimuthal angle $\alpha$ for chosen values of the polar angle $\beta$, computed by optimizing the remaining six coordinates $\left\{d, d_{\mathrm{H}}, d_{\mathrm{N}}, R, \theta, \gamma\right\}$. The curves shown are measured relative to the energy of the optimum equilibrium configuration.

$\mathrm{NH}_{3}-\mathrm{H}_{2}$ by exploring paths along selected angular coordinates or coordinate pairs with optimization of the remaining coordinates at each point. The numerous minimizations are most economically done with modest basis set sizes whose reliability has been assessed by the procedures outlined in the previous sections.

The variation of the CCSD(T)/aug-cc-pVTZ potential 


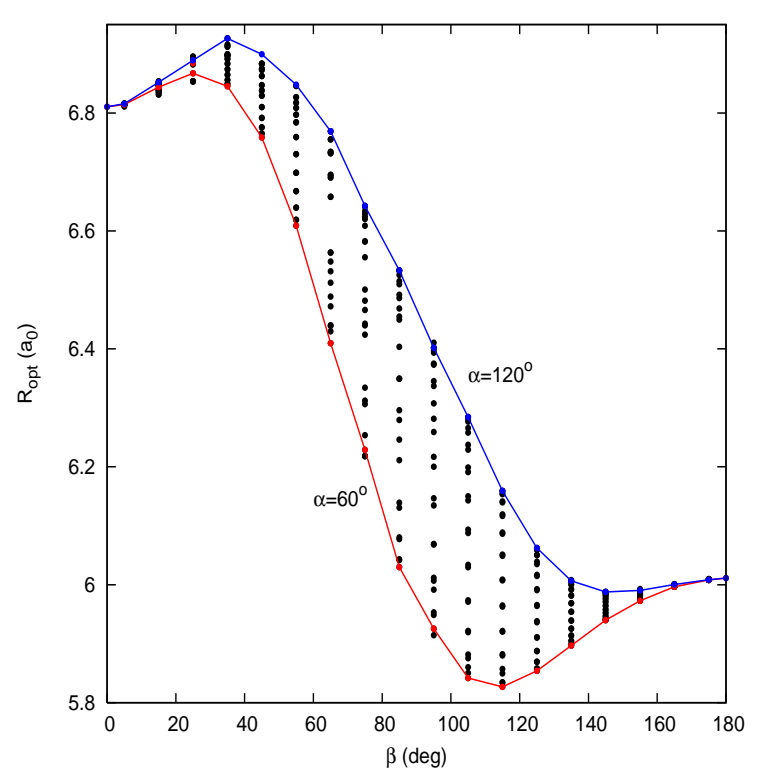

FIG. 5: Optimum values of the intermolecular distance $R$, corresponding to the $\operatorname{CCSD}(\mathrm{T}) /$ aug-cc-pVTZ results shown in Fig. 4.

energy along the azimuthal angle $\alpha$ for chosen values of the polar angle $\beta$ is shown in Fig. 4 . The two angles, $\alpha$ and $\beta$, considered first specify the orientation of the intermolecular vector $\mathbf{R}$ with respect to the ammonia subunit, see Fig. 1. For a given $(\beta, \alpha)$ pair, the complex geometry was optimized for pyramidal ammonia with respect to the remaining six coordinates, $\left\{d, d_{\mathrm{H}}, d_{\mathrm{N}}, R, \theta, \gamma\right\}$.

Two types of energy profiles along the angle $\alpha$ may be readily identified in Fig. 4. The paths along the azimuthal angle $\alpha$ computed for $\beta$ larger than $75^{\circ}$ exhibit a single minimum at $\alpha=60^{\circ}$. For $\beta$ smaller than $75^{\circ}$, the profiles exhibit two symmetry equivalent minima for $\alpha$ of $0^{\circ}$ and $120^{\circ}$. The potential energy profile calculated for $\beta=75^{\circ}$ possesses three nearly equivalent minima, which are separated by very small barriers of ca. $8 \mathrm{~cm}^{-1}$. Note that $\alpha=60^{\circ}$ corresponds to the intermolecular vector $\mathbf{R}$ spatially oriented between two hydrogen atoms of ammonia (called a middle position here), whereas $R$ is placed above one of the three $\mathrm{H}$ atoms of $\mathrm{NH}_{3}$ for $\alpha=0,120^{\circ}$ (called a NH line position here).

Along the optimized energy profiles of Fig. 4, the most prominent changes are found for the intermolecular distance $R$. The corresponding variations in $R_{o p t}$ are displayed in Fig. 5. The minimum and maximum values of $R_{\text {opt }}$ for a given polar angle $\beta$ are seen there for $\alpha=60^{\circ}$ and $\alpha=120^{\circ}$, respectively. For e.g. $\beta=85^{\circ}$, the distance $R_{\text {opt }}$ varies between $6.03 \mathrm{a}_{0}\left(\right.$ for $\left.\alpha=60^{\circ}\right)$ and 6.53 $\mathrm{a}_{0}$ (for $\alpha=0^{\circ}, 120^{\circ}$ ). This $R_{o p t}$ variation is associated with an energy change of only $18 \mathrm{~cm}^{-1}$.

Along the energy profiles of Fig. 4, the three distances $d_{\mathrm{H}}, d_{\mathrm{N}}$, and $d$ vary within about $0.003 \mathrm{a}_{0}, 0.006 \mathrm{a}_{0}$, and $0.004 \mathrm{a}_{0}$, respectively. These small changes of $d_{\mathrm{H}}, d_{\mathrm{N}}$, and $d$ indicate that the monomers stay to a great extent rigid during the orbital motion of the vector $\mathbf{R}$ around

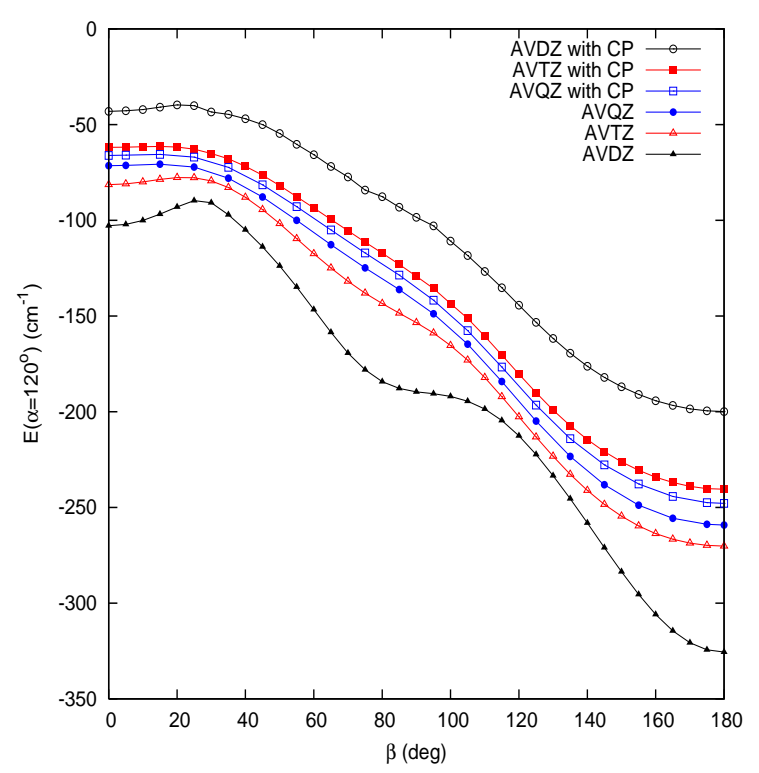

FIG. 6: Energy profiles along the polar angle $\beta$ computed for the rigid monomers and the angle $\alpha$ equal to $120^{\circ}$. The curves are obtained by the two-dimensional energy optimization with respect to $R$ and $\theta$, assuming $\gamma=0^{\circ}$. The zero energy is defined as the energy of infinitely separated monomers.

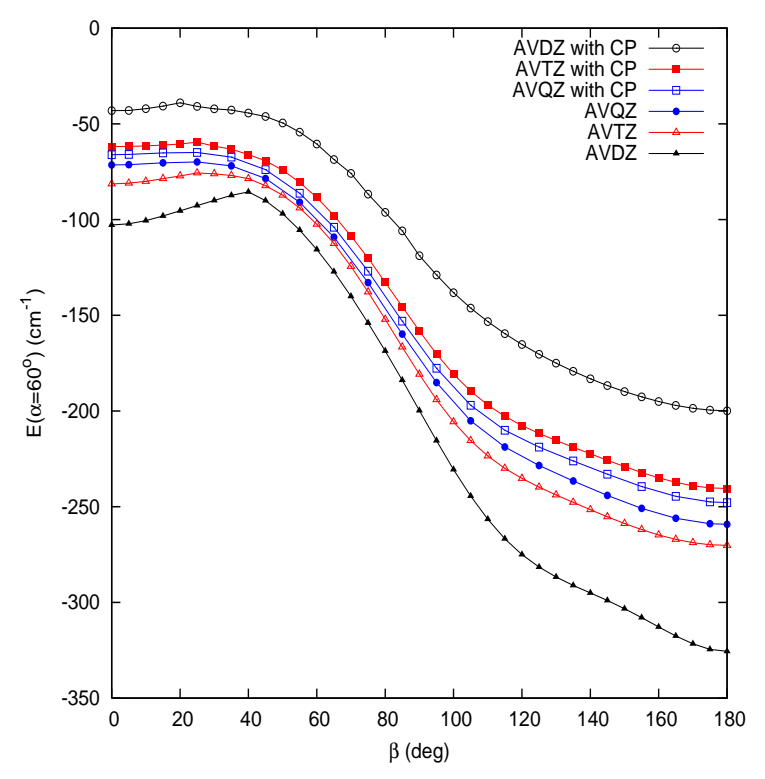

FIG. 7: Same as Fig. 6, only for $\alpha$ equal to $60^{\circ}$.

the $\mathrm{NH}_{3}$ moiety.

Since the energy profiles in Fig. 4 readily show two distinct regions around $\alpha=60^{\circ}$ and $\alpha=120^{\circ}$, we have decided to examine these subspaces in more detail using the $\operatorname{CCSD}(\mathrm{T}) /$ aug-cc-pVXZ approach for $\mathrm{X}=\mathrm{D}, \mathrm{T}, \mathrm{Q}$. The energy profiles along the coordinate $\beta$, computed for rigid monomers at their respective optimum geometry of TableII, are displayed for $\alpha=120^{\circ}$ and $\alpha=60^{\circ}$ in Figs. 6 and 7, respectively. The optimization was performed with respect to $R$ and $\theta$, assuming $\gamma=0^{\circ}$. Note 


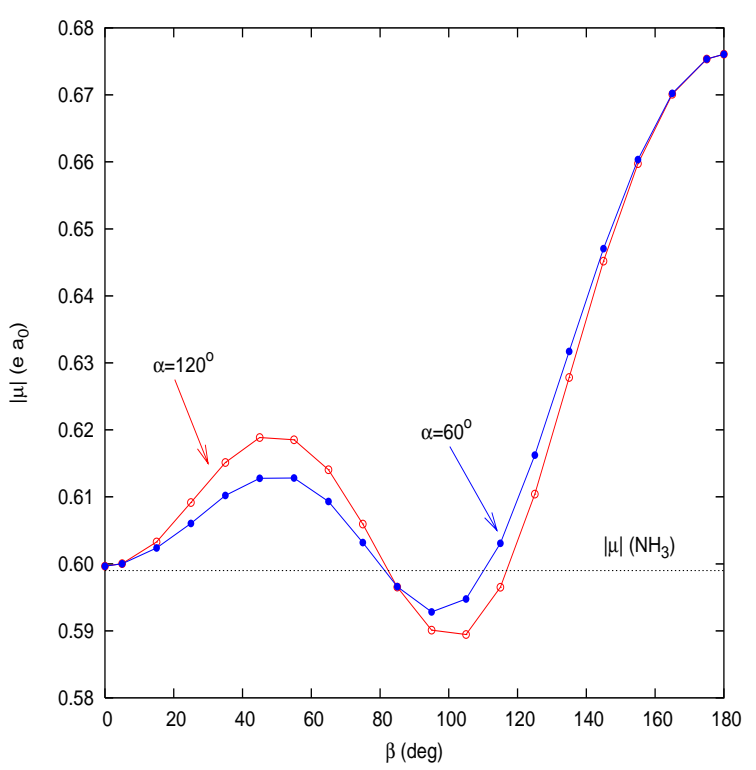

FIG. 8: Variation of the MP2 dipole moment $|\mu|$ along the CCSD(T)/aug-cc-pVQZ optimum energy paths shown in Figs. 6 and 7. The horizontal line at $|\mu|=0.599 e a_{o}$ shows the corresponding value for the dipole moment of free ammonia.

that all points on the energy profiles in Figs. 6 and 7 correspond to electronically bound arrangements of the complex.

At the double-zeta level, the CP uncorrected profiles in Figs. 6 and 7 show an additional minimum for the configuration involving an inverted ammonia subunit (when $\beta=0^{\circ}$ ) with additional flattening around $\beta=90^{\circ}$ for $\alpha=120^{\circ}$. The CP uncorrected and CP corrected profiles from the aug-cc-pVTZ and aug-cc-pVQZ calculations are, however, less structured, exhibiting a single minimum at $\beta=180^{\circ}$. The optimum $\operatorname{CCSD}(\mathrm{T}) /$ aug-ccpVQZ arrangements with $\beta=0^{\circ}$ lie 188 and $182 \mathrm{~cm}^{-1}$ above the minimum $\beta=180^{\circ}$ arrangements in the $\mathrm{CP}$ uncorrected and $\mathrm{CP}$ corrected situation, respectively.

Figure 8 shows the variation of the absolute value of the dipole moment

$$
|\mu|=\sqrt{\mu_{x}^{2}+\mu_{y}^{2}+\mu_{z}^{2}} .
$$

along the optimum $\beta$ paths shown in Figs. 6 and 7 and in relation to the permanent dipole moment of ammonia.

The energy difference $\Delta E_{\alpha}$,

$$
\Delta E_{\alpha}=E_{\text {opt }}\left(\alpha=120^{\circ}\right)-E_{\text {opt }}\left(\alpha=60^{\circ}\right),
$$

between the optimum $\alpha=120^{\circ}$ and optimum $\alpha=60^{\circ}$ configurations as a function of the angle $\beta$ is seen in Fig. 9, where the CP uncorrected aug-cc-pVXZ results for $\mathrm{X}=\mathrm{D}, \mathrm{T}, \mathrm{Q}$ are used. The $\Delta E_{\alpha}$ values obtained from $\mathrm{CP}$ uncorrected and CP corrected $\operatorname{CCSD}(\mathrm{T}) /$ aug-cc-pVXT energies with $\mathrm{X}=\mathrm{T}$ and $Q$ are compared in Fig. 10 . There, $\Delta E_{\alpha}=0$ is seen for $\beta \approx 85^{\circ}, 75^{\circ}$, and $70^{\circ}$ for $\mathrm{X}=\mathrm{D}, \mathrm{T}$ and $\mathrm{Q}$, respectively. For $\mathrm{CP}$ uncorrected and

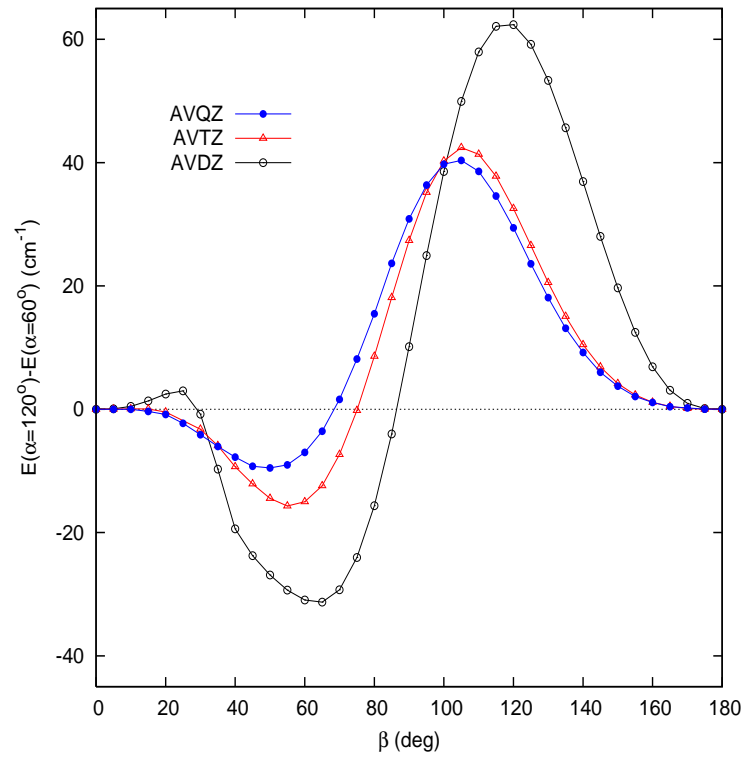

FIG. 9: Energy difference $E\left(\alpha=120^{\circ}\right)-E\left(\alpha=60^{\circ}\right)$ as a function of the angle $\beta$, obtained from the $\mathrm{CP}$ uncorrected CCSD $(\mathrm{T}) /$ aug-cc-pVXZ energy profiles $(\mathrm{X}=\mathrm{D}, \mathrm{T}$, and $\mathrm{Q})$, shown in Figs. 6 and 7.

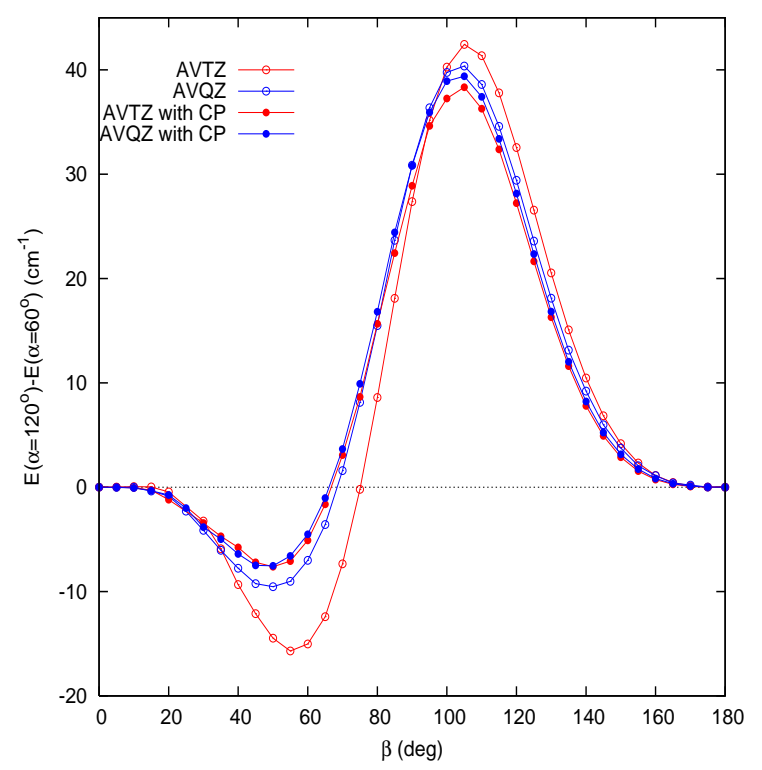

FIG. 10: Same as Fig. 9, only from the CP uncorrected and CP corrected CCSD(T)/aug-cc-pVXZ energies obtained using the basis sets of $\mathrm{X}=\mathrm{T}$ and $\mathrm{Q}$.

$\mathrm{CP}$ corrected aug-cc-pVTZ results in Fig. 10, we have $\Delta E_{\alpha}=0$ at respectively $\beta \approx 75^{\circ}$ and $\beta \approx 65^{\circ}$. At the quadruple-zeta level, the difference between zeroes of $\Delta E_{\alpha}$ from the $\mathrm{CP}$ uncorrected and $\mathrm{CP}$ corrected calculations is small (in order of a degree). One may note that the $\mathrm{N}-\mathrm{H}$ distance vector encloses a $\beta$ angle of about $67^{\circ}$ with the $C_{3}$ symmetry axis of ammonia.

As seen in Figs. 4, 9, and 10, the preferential position of the intermolecular $\mathbf{R}$ vector is characterized by $\alpha=$ 


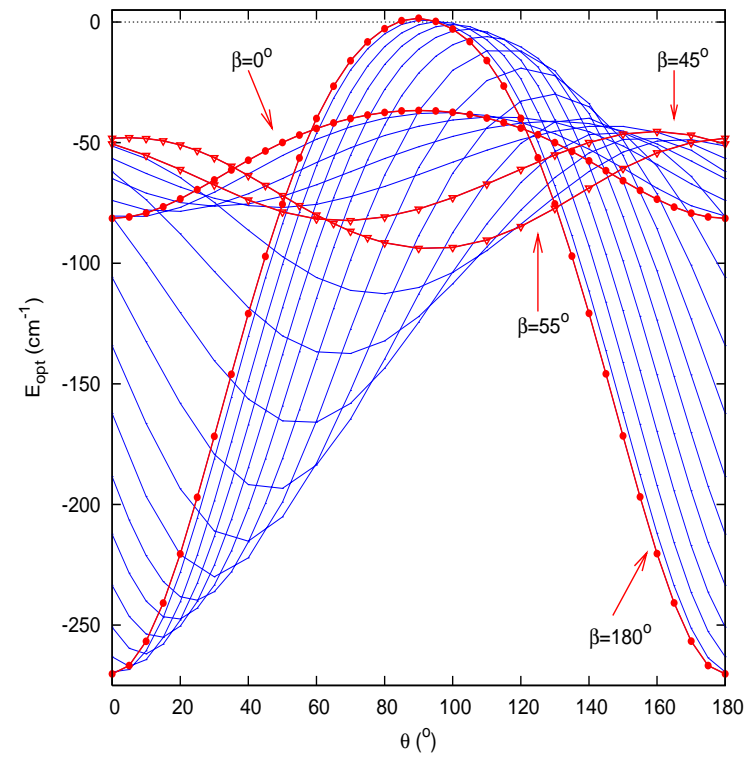

FIG. 11: Variation of the CCSD(T)/aug-cc-pVTZ potential energy along the polar angle $\theta$ for selected $\beta$ orientations of the intermolecular vector $\mathbf{R}$. The curves shown are computed for rigid monomers by the one-dimensional optimization with respect to $R$, assuming $\alpha=60^{\circ}$ and $\gamma=0^{\circ}$.

$60^{\circ}$ (middle positions) over a wide range of $\beta$ values, $\beta \in\left(70^{\circ}, 180^{\circ}\right)$. For $\beta \in\left(0^{\circ}, 70^{\circ}\right)$, the vector $\mathbf{R}$ assumes positions described by $\alpha=0,120^{\circ}$ (NH line positions). The maximum CCSD (T)/aug-cc-pVQZ energy difference $\Delta E_{\alpha}$ in Fig. 10 is about $40 \mathrm{~cm}^{-1}$ and $9 \mathrm{~cm}^{-1}$ at $\beta \approx 105^{\circ}$ and $\beta \approx 45^{\circ}$ for a lower energy $\alpha=60^{\circ}$ and $\alpha=120^{\circ}$ configuration, respectively.

The orientation of molecular hydrogen around ammonia is described by two angles, $\theta$ and $\gamma$, as shown in Fig. 1. The properties of the potential energy surface with respect to these two angles are described next.

In Fig.11, we first consider the variation of the $\operatorname{CCSD}(\mathrm{T}) /$ aug-cc-pVTZ energy with the polar angle $\theta$ for selected orientations of the intermolecular vector $\mathbf{R}$ with respect to the $C_{3 v}$ axis of $\mathrm{NH}_{3}$. In these calculations, rigid monomers are oriented such that $\alpha=60^{\circ}$ and $\gamma=0^{\circ}$ are assumed. The calculations have been performed by optimizing the intermolecular distance $R$.

Molecular hydrogen is aligned $\left(\theta=0,180^{\circ}\right)$ with the intermolecular vector $\mathbf{R}$ for $\beta=180^{\circ}$ (equilibrium) and $\beta=0^{\circ}$ (inverted $\mathrm{NH}_{3}$ ). The minima with $\beta=180^{\circ}$ and $\beta=0^{\circ}$ in Fig. 11 are lying ca. 270 and $45 \mathrm{~cm}^{-1}$ lower than the corresponding T-shaped forms $\left(\theta=90^{\circ}\right)$, respectively. In the course of orbiting of $\mathbf{R}$ about the $\mathrm{NH}_{3}$ moiety, the $\mathrm{H}_{2}$ subunit preferentially assumes bent positions, i.e. $\theta \neq 0,180^{\circ}$. The minimum $\theta$ value of $90^{\circ}$ is seen in Fig. 11 for the energy profile computed for $\beta=55^{\circ}$.

The internal rotation of $\mathrm{H}_{2}$ described with the help of the angles $\theta$ and $\gamma$ is graphically displayed in Figs. 12 and 13 for $\beta=180^{\circ}, 0^{\circ}$ (equilibrium, inverted $\mathrm{NH}_{3}$ ) and for $\beta=67.2^{\circ}$ (NH line position), respectively. Molecular

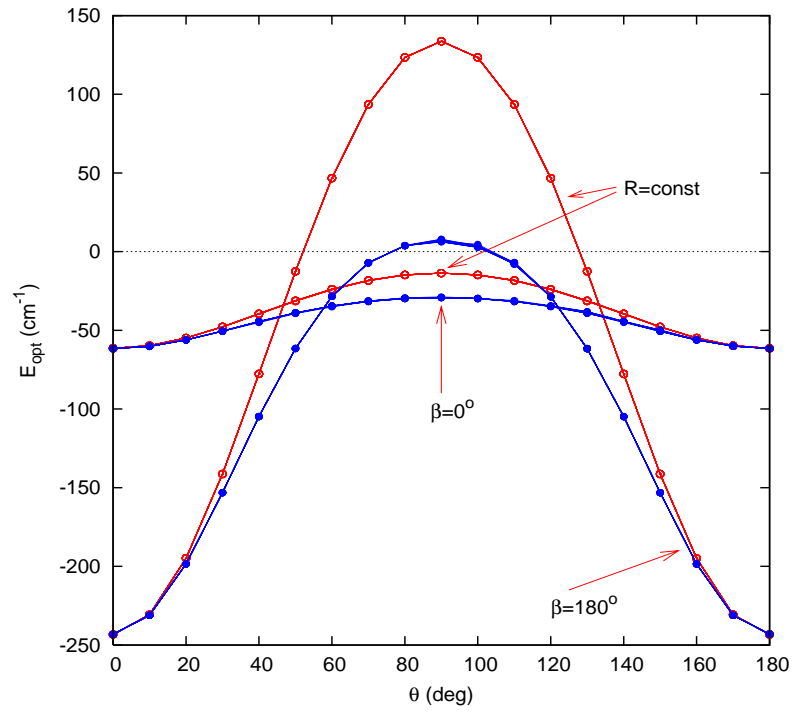

FIG. 12: CP corrected CCSD(T)/aug-cc-pVTZ energies computed for a chosen angular $(\theta, \gamma)$ grid and $\beta=180^{\circ}, 0^{\circ}$ assuming rigid monomers. The $\mathrm{H}-\mathrm{H}$ distance $d$ of $\mathrm{H}_{2}$ is optimized here. The lower of the two curves shown for a given $\beta$ value is computed by optimizing also the intermolecular distance $R$.

hydrogen clearly exhibits a somewhat hindered internal rotation in arrangements near the global minimum at $\beta=180^{\circ}$, whereas in those with $\beta=0^{\circ} \mathrm{H}_{2}$ performs nearly free rotation encountering a small barrier of only $25 \mathrm{~cm}^{-1}$, Fig. 12 .

For the intermolecular vector $R$ enclosing with the $C_{3 v}$ axis of $\mathrm{NH}_{3}$ the same angle as $\mathrm{N}-\mathrm{H}$ bond-distance vectors of pyramidal $\mathrm{NH}_{3}$, molecular hydrogen preferentially assumes a T-shaped orientation described by $\theta \approx 90^{\circ}$. This is seen in Fig. 13 , where we show the $\mathrm{CP}$ corrected CCSD(T)/aug-cc-pVTZ energies computed for rigid monomers by optimizing both the intermolecular distance $R$ and the $\mathrm{H}-\mathrm{H}$ distance in $\mathrm{H}_{2}$. For $\beta=67.2^{\circ}$, the internal rotation of $\mathrm{H}_{2}$ about $\mathrm{NH}_{3}$ is also somewhat hindered around $\theta=90^{\circ}$ for $\alpha=120^{\circ}$ and $\theta=75,105^{\circ}$ for $\alpha=60^{\circ}$. One may additionally note that the potential energy is only weakly anisotropic along the azimuthal angle $\gamma$ for $\beta=67.2^{\circ}$ and $\alpha=120^{\circ}$ in Fig. 13 and for $\beta=180,0^{\circ}$ in Fig. 12 .

\section{CONCLUSION}

The intermolecular potential energy surface for the van der Waals complex between ammonia and molecular hydrogen has been studied by means of high-level $a b$ initio calculations employing several families of one-electron basis set families. The potential energy surface becomes progressively less structured as the basis set size is increased. The effect of the basis set superposition error on the interaction energy has been analysed and is found to become very small at the aug-cc-pVQZ level. At this level the weak local minimum with $\mathrm{H}_{2}$ on the hydrogen 

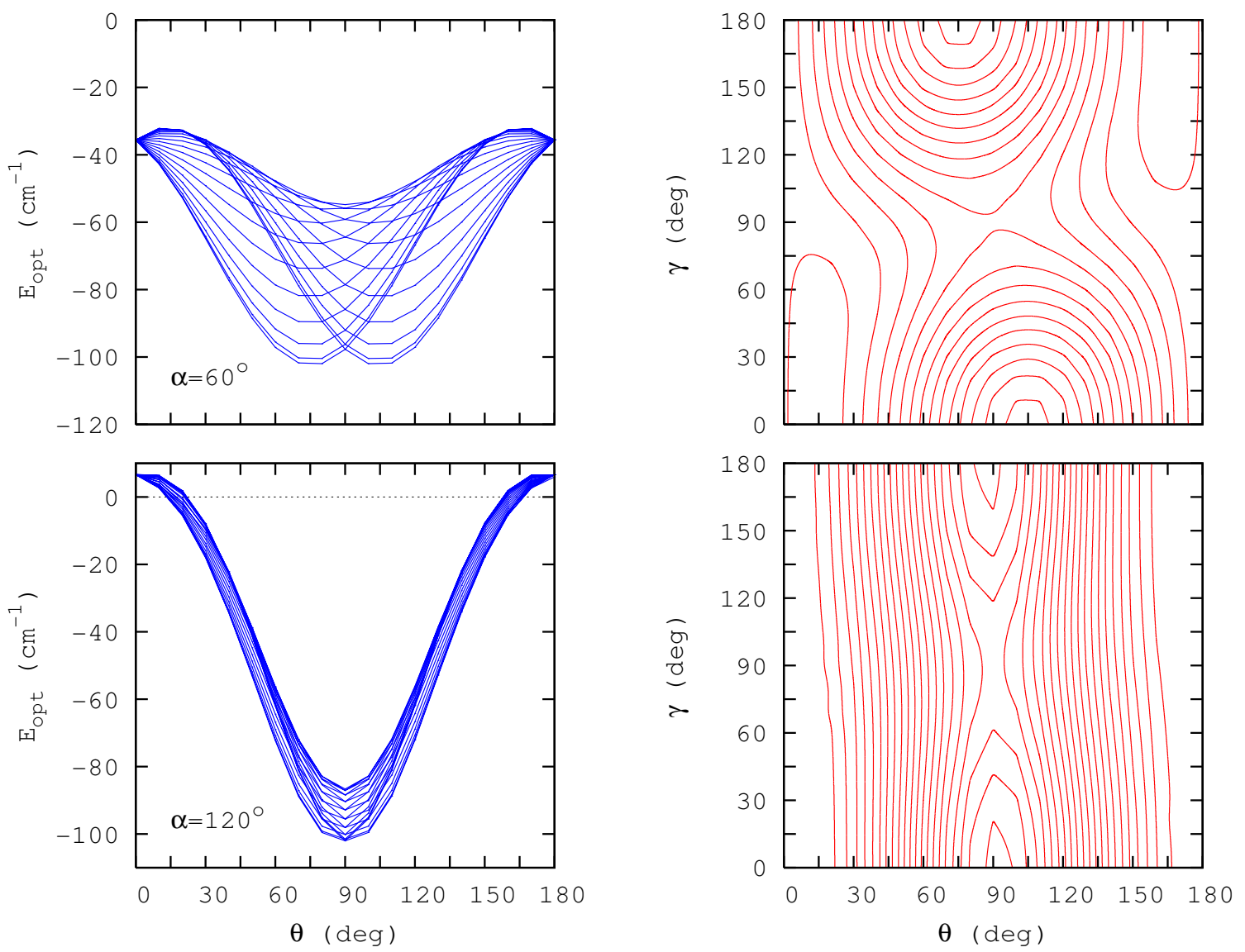

FIG. 13: CP corrected CCSD(T)/aug-cc-pVTZ potential energies computed for a chosen angular $(\theta, \gamma)$ grid, employing rigid monomers and $\mathrm{NH}$ line oriented molecular hydrogen $\left(\beta=67.2^{\circ}\right)$. The intermolecular distance $R$ and $\mathrm{H}-\mathrm{H}$ distance $d$ of $\mathrm{H}_{2}$ are optimized. Both $\alpha=60^{\circ}$ situation (upper figures) and $\alpha=120^{\circ}$ situation (lower figures) are considered. The variation of the potential energy along the angle $\theta$ for chosen values of $\gamma$ are shown on the left hand side. The two-dimensional $(\theta, \gamma)$ maps shown on the right hand side are obtained with the help of a spline routine. Contour lines are drawn in intervals of $5 \mathrm{~cm}^{-1}$ with the first contour placed at $-100 \mathrm{~cm}^{-1}$ for both $\alpha=60^{\circ}$ and $\alpha=120^{\circ}$.

side of the $\mathrm{NH}_{3}$ symmetry axis disappears in favor of a large flat region of the potential with respect to $\mathrm{H}_{2}$ position and orientation.

The only remaining minimum is found to correspond to $\mathrm{H}_{2}$ on the $C_{3 v}$ axis near the nitrogen atom. This minimum is bound by $D_{e}=253 \mathrm{~cm}^{-1}$ for $\mathrm{H}_{2}$ aligned with the $C_{3 v}$ axis. The potential rises steeply for other $\mathrm{H}_{2}$ orientations indicating somewhat hindered internal rotation. The analysis of minimum energy paths along selected angular coordinate combinations shows the presence of pronounced angular-radial coupling (Figs. 5 and 12). A global minimum with $\mathrm{H}_{2}$ near a lone pair corresponds to the situation found in $\mathrm{H}_{2} \mathrm{O}-\mathrm{H}_{2}{ }^{9,17}$ but differs from the best minimum found for $\mathrm{HF}-\mathrm{H}_{2}{ }^{42}$.

Anharmonic vibrational corrections to the binding energy are expected to be very large and $D_{0}$ is probably only a small fraction of $D_{e}$. $D_{0}$ values as small as $20 \%$ of $D_{e}$ are known from theoretical work for complexes between molecules and helium ${ }^{43}$ and a combined experimental and theoretical analysis of the $\mathrm{NH}-\mathrm{H}_{2}$ complex ${ }^{44}$ has given $D_{e}=116.3 \mathrm{~cm}^{-1}$ and $D_{0}=32 \pm 2 \mathrm{~cm}^{-1}$.

The topographical features of the $\mathrm{NH}_{3}-\mathrm{H}_{2}$ interaction revealed by the present explorations supply important input for the construction of an analytical representation of the full 5-dimensional surface whose construction is under way. This surface will allow the immediate determination of $D_{0}$ by quantum Monte Carlo and/or variational methods ${ }^{45}$.

\section{Acknowledgments}

MM would like to thank Prof. Peter Botschwina for his kind and permanent support during her Habilitation thesis at the University of Göttingen. 
* Corresponding author. Tel.: +33160957317; fax: +33 160 957320; Electronic address: mladenov@univ-mlv. fr

$\dagger$ Electronic address: lewerenz@univ-mlv.fr

₹ Electronic address: cilpa@univ-mlv.fr

$\S$ Electronic address: rosmus@univ-mlv.fr

ब Electronic address: chambaud@univ-mlv.fr

1 S. Green, J. Chem. Phys. 73 (1980) 2740.

2 A. Offer and D. R. Flower, J. Chem. Soc., Faraday Trans. 86 (1990) 1659.

3 C. Rist, M. H. Alexander and P. Valiron, J. Chem. Phys. 98 (1993) 4662.

4 A. Moroz, R. L. Sweany and S. L. Whittenburg, J. Phys. Chem. 94 (1990) 1352.

${ }^{5}$ M. E. Jacox and W. E. Thompson, J. Chem. Phys. 124 (2006) 204304.

6 C. M. Lovejoy, D. D. Nelson, Jr. and D. J. Nesbitt, J. Chem. Phys. 87 (1987) 5621.

7 C. M. Lovejoy, D. D. Nelson, Jr. and D. J. Nesbitt, J. Chem. Phys. 89 (1988) 7180.

8 M. J. Weida and D. J. Nesbitt, J. Chem. Phys. 110 (1999) 156.

9 M. P. Hodges, R. J. Wheatley, G. K. Schenter and A. H. Harvey, J. Chem. Phys. 120 (2004) 710.

10 D. R. Willey, R. E. Timlin, Jr., C. D. Ruggiero and I. A. Sulai, J. Chem. Phys. 120 (2004) 129.

11 M. Behrens, U. Buck, R. Fröchtenicht, M. Hartmann, F. Huisken and F. Rohmund, J. Chem. Phys. 109 (1998) 5914.

12 M. E. Fajardo and S. Tam, J. Chem. Phys. 115 (2001) 6807.

13 G. A. Olah, D. J. Donovan, J. Shen and G. J. Klopman, J. Am. Chem. Soc. 97 (1975) 3559.

14 M. P. Hodges and R. J. Wheatley, J. Chem. Phys. 114 (2001) 8836.

15 T. R. Phillips, S. Maluendes, A. D. McLean and S. Green, J. Chem. Phys. 101 (1994) 5824.

16 T. R. Phillips, S. Maluendes and S. Green, J. Chem. Phys. 102 (1995) 6024.

17 A. Faure, P. Valiron, M. Wernli, L. Wiesenfeld, C. Rist, J. Noga and J. Tennyson, J. Chem. Phys. 122 (2005) 221102.

18 C. Hampel, K. A. Peterson and H.-J. Werner, Chem. Phys. Lett. 190 (1992) 1, and references therein.

19 M. J. O. Deegan and P. J. Knowles, Chem. Phys. Lett. 227 (1994) 321.

20 R. A. Kendall, T. H. Dunning and R. J. Harrison, J. Chem. Phys. 96 (1992) 6796.

21 T. J. Lee and P. R. Taylor, Int. J. Quant. Chem. Symp. 23 (1989) 199.

22 MOLPRO, version 2006.1, a package of ab initio programs,
H.-J. Werner, P. J. Knowles, R. Lindh, F. R. Manby, M. Schütz, and others, see http://www.molpro.net.

${ }^{23}$ F. Eckert, P. Pulay and H.-J. Werner, Theor. Chem. Acc. 100 (1998) 21.

24 S. F. Boys and F. Bernardi, Mol. Phys. 19 (1970) 553.

25 D. Feller, J. Chem. Phys. 96 (1992) 6104.

26 J. J. Novoa, M. Planas and M. H. Whangbo, Chem. Phys. Lett. 225 (1994) 240.

27 M. Mladenović, J. Chem. Phys. 113 (2000) 10524.

28 G. Herzberg, Molecular Spectra \& Molecular Structure Vol. III, Electronic Spectra and Electronic Structure of Polyatomic Molecules (corrected reprint of 1966 edition), (Krieger, Malabar FL, 1991).

29 T. N. Olney, N. M. Cann, G. Cooper and C. E. Brion, Chem. Phys. 223 (1997) 59.

30 A. D. McLean and M. Yoshimine, J. Chem. Phys. 45 (1966) 3676.

31 P. Piecuch, V. Špirko and J. Paldus, J. Chem. Phys. 105 (1996) 11068.

32 S. G. Kukolich and I. Casleton, Chem. Phys. Lett. 18 (1973) 408.

33 A. J. Stone, Intermolecular Forces, (Clarendon Press, Oxford, 1996).

34 J. Rheinecker and J. M. Bowman, J. Chem. Phys. 125 (2006) 133206.

35 W. Kołos and L. Wolniewicz, J. Chem. Phys. 46 (1967) 1426 .

36 I. S. Lim, P. Botschwina, R. Oswald, V. Barone, H. Stoll and P. Schwerdtfeger, J. Chem. Phys. 127 (2007) 104313.

37 A. Halkier and P. R. Taylor, Chem. Phys. Lett. 285 (1998) 133.

38 N. F. Ramsey, Molecular Beams, (Oxford University Press, Oxford, 1956).

${ }^{39}$ K. P. Huber and G. Herzberg, Molecular Spectra \& Molecular Structure Vol. IV. Constants of Diatomic Molecules, (Prentice-Hall, Englewood Cliffs, NJ, 1979).

40 D. E. Woon and T. H. Dunning, Jr., J. Chem. Phys. 103 (1995) 4572.

41 T. Helgaker, W. Klopper, H. Koch and J. Noga, J. Chem. Phys. 106 (1997) 9639.

42 D. E. Bernholdt, S.-Y. Liu and C. E. Dykstra, J. Chem. Phys. 85 (1986) 5120.

43 D. Blume, M. Lewerenz, F. Huisken and M. Kaloudis, J. Chem. Phys. 105 (1996) 8666.

${ }^{44}$ W. M. Fawzy, G. Kerenskaya and M. C. Heaven, J. Chem. Phys. 122 (2005) 144318.

45 M. Mladenović and M. Lewerenz, Chem. Phys. Lett. 321 (2000) 135. 